\title{
Robot Yardımlı Laparoskopik Radikal Prostatektomide Tam Kontinans İçin Neler Yapılmalı?
}

\author{
Hasan Hüseyin Tavukçu, Ömer Aytaç, Fatih Atuğ
}

İstanbul Bilim Üniversitesi, Tıp Fakültesi, Üroloji anabilim Dalı, İstanbul

Giriş

çık radikal prostatektomi (ARP) sonrası idrar kaçırma,
bostaların yaşam kalitesini ve hasta memnuniyetini
bozan en önemli etkenlerden biri olarak karşımıza çıkmak-
tadır (1). Yıllar boyunca radikal prostatektomi ameliyatla-
rındaki gelişmelere rağmen ARP ve laparoskopik radikal
prostatektomi (LRP) serilerinde \%60 ile \%95 arasında konti-
nans oranları bildirilmiştir (2,3,4). Robotik teknolojinin optik
büyütme, üç boyutlu görüntü ve 7 derece harekete imkan
tanıyan robotik kolları; kontinans ve erektil fonksiyonda etki-
li anatomik noktaların korunmasına ve perioperatif komp-
likasyonların azalmasına imkan sağlamaktadır. İlk yıllarda
yapılan robot yardımlı laparoskopik radikal prostatektomi
(RYLP) operasyonları, açı ve konvansiyonel laparoskopik seriler ile karşılaştırıldığında, kontinans iyileşmesi açısından anlamlı farklılık ve teknikler arasında birbirlerine üstünlükler görülmemesine karşın, son yıllarda bildirilen RYLP serilerinde, ameliyat tekniklerindeki gelişmelere paralel olarak kontinans oranlarında oldukça anlamlı iyileşmeler rapor edilmektedir (2). Bu derlememizde RYLP' de tam kontinans elde edilmesi için neler yapılması gerektiğini literatür eşliğinde tartışacağız.

\section{RYLP Sonrası İdrar Kaçırma Oranları ve İdrar Kaçırmayı Öngören Özellikler}

Güncel RYLP serilerindeki kontinans durumu Tablo 1'de verilmiştir. Tablo'daki çalışmalarda görüldüğü üzere 12 ay sonunda hiç ped kullanmama temel alındığında kontinans

Tablo 1: RYLP sonrası kontinans oranları

\begin{tabular}{|c|c|c|c|c|c|c|c|c|}
\hline $\begin{array}{l}\text { Yazar/ } \\
\text { Çalışma yılı }\end{array}$ & $\begin{array}{l}\text { Vaka } \\
\text { sayısı }\end{array}$ & $\begin{array}{l}\text { Çalışma } \\
\text { dizaynı }\end{array}$ & $\begin{array}{l}\text { Kontinans } \\
\text { tanimlama }\end{array}$ & Bilgi toplama & $\begin{array}{l}\text { 3. ay } \\
\text { kontinans } \\
(\%)\end{array}$ & $\begin{array}{l}\text { 6. ay } \\
\text { kontinans } \\
(\%)\end{array}$ & $\begin{array}{l}\text { 12. ay } \\
\text { kontinans } \\
(\%)\end{array}$ & $\begin{array}{l}24-36 \text {. ay } \\
\text { kontinans } \\
(\%)\end{array}$ \\
\hline $\begin{array}{l}\text { Finley(5) } \\
2009\end{array}$ & 666 & Prospektif & 0 ped & Valide anket & 69 & - & - & - \\
\hline $\begin{array}{l}\text { Lee(6) } \\
2010\end{array}$ & 107 & Prospektif & 0 ped & Belirtilmemiş & - & - & 91 & - \\
\hline $\begin{array}{l}\text { Novara(7) } \\
2010\end{array}$ & 308 & Prospektif & 0 ped & Valide anket & - & - & 90 & - \\
\hline $\begin{array}{l}\text { Shikanov(8) } \\
2010\end{array}$ & 1436 & Prospektif & 0 ped & Valide anket & - & - & 69 & - \\
\hline $\begin{array}{l}\text { Martin(9) } \\
2011\end{array}$ & 315 & Prospektif & 0 ped & Valide anket & - & - & 78 & - \\
\hline $\begin{array}{l}\text { Patel(10) } \\
2011\end{array}$ & 1111 & Prospektif & 0 ped & Valide anket & 86 & 94 & 96 & - \\
\hline $\begin{array}{l}\text { Xylinas(11) } \\
2010\end{array}$ & 500 & Prospektif & 0 ped & Valide anket & - & - & 78 & 88 \\
\hline $\begin{array}{l}\operatorname{Link}(12) \\
2008\end{array}$ & 1847 & Prospektif & $\begin{array}{l}0-1 \text { güvenlik } \\
\text { pedi }\end{array}$ & Görüşme & - & - & 92.5 & - \\
\hline $\begin{array}{l}\text { Greco(13) } \\
2009\end{array}$ & 180 & Prospektif & $\begin{array}{l}0-1 \text { güvenlik } \\
\text { pedi }\end{array}$ & Anket & 65 & 79 & 89 & - \\
\hline $\begin{array}{l}\text { Murphy(14) } \\
2009\end{array}$ & 400 & Prospektif & $\begin{array}{l}0-1 \text { güvenlik } \\
\text { pedi }\end{array}$ & Belirtilmemiş & - & - & 91 & 95 \\
\hline $\begin{array}{l}\text { Samadi(15) } \\
2010\end{array}$ & 1181 & Prospektif & $\begin{array}{l}0-1 \text { güvenlik } \\
\text { pedi }\end{array}$ & Belirtilmemiş & - & - & 91.3 & - \\
\hline
\end{tabular}


Tablo 2: RYLP sonrası komplike vakalarda ve cerrahi tecrübede kontinans oranları

\begin{tabular}{|c|c|c|c|c|c|c|c|c|}
\hline $\begin{array}{l}\text { Yazar/ } \\
\text { Çalışma yılı }\end{array}$ & $\begin{array}{l}\text { Vaka } \\
\text { say1sı }\end{array}$ & $\begin{array}{l}\text { Çalışma } \\
\text { dizaynı }\end{array}$ & $\begin{array}{l}\text { Kontinans } \\
\text { tanımlama }\end{array}$ & $\begin{array}{l}\text { Bilgi } \\
\text { toplama }\end{array}$ & $\begin{array}{l}\text { 3. ay } \\
\text { kontinans } \\
(\%)\end{array}$ & $\begin{array}{l}\text { 6. ay } \\
\text { kontinans } \\
(\%)\end{array}$ & $\begin{array}{l}\text { 12. ay } \\
\text { kontinans } \\
(\%)\end{array}$ & $\begin{array}{l}\text { 24-36. ay } \\
\text { kontinans } \\
(\%)\end{array}$ \\
\hline $\begin{array}{l}\operatorname{Link}(12) \\
2008\end{array}$ & $\begin{array}{l}\text { PB }<30 \mathrm{~cm}^{3}, 69 \\
\text { PB } 30-49 \mathrm{~cm}^{3}, 883 \\
\text { PB } 50-69 \mathrm{~cm}^{3}, 568 \\
\text { PB } \geq 70 \mathrm{~cm}^{3}, 327\end{array}$ & Prospektif & $\begin{array}{l}0-1 \text { güvenlik } \\
\text { pedi }\end{array}$ & Görüşme & - & - & $\begin{array}{l}93 \\
94 \\
93 \\
88\end{array}$ & - \\
\hline $\begin{array}{l}\text { Skolarus(16) } \\
2010\end{array}$ & $\begin{array}{l}\mathrm{PB}<50 \mathrm{~cm}^{3}, 582 \\
\text { PB } 50-100 \mathrm{~cm}^{3}, 279 \\
\mathrm{~PB} \geq 100 \mathrm{~cm}^{3}, 24\end{array}$ & - & 0 ped & $\begin{array}{l}\text { Valide } \\
\text { anket }\end{array}$ & $\begin{array}{l}62 \\
49 \\
44\end{array}$ & - & - & - \\
\hline $\begin{array}{l}\text { Wiltz(17) } \\
2009\end{array}$ & $\begin{array}{l}\text { VKİ }<25,216 \\
\text { VKİ 25-30, } 464 \\
\text { VKİ }>30,265\end{array}$ & Prospektif & 0 ped & $\begin{array}{l}\text { Valide } \\
\text { anket }\end{array}$ & $\begin{array}{l}33 \\
31 \\
28\end{array}$ & $\begin{array}{l}58 \\
52 \\
50\end{array}$ & $\begin{array}{l}70 \\
68 \\
57\end{array}$ & $\begin{array}{l}75 \\
71 \\
57\end{array}$ \\
\hline $\begin{array}{l}\text { Zorn(18) } \\
2009\end{array}$ & $\begin{array}{l}1-300 \text { vaka } \\
301-500 \text { vaka } \\
501-700 \text { vaka }\end{array}$ & Prospektif & 0 ped & $\begin{array}{l}\text { Valide } \\
\text { anket }\end{array}$ & $\begin{array}{l}26 \\
30 \\
41\end{array}$ & $\begin{array}{l}47 \\
51 \\
64\end{array}$ & $\begin{array}{l}62 \\
65 \\
75\end{array}$ & - \\
\hline $\begin{array}{l}\text { Samadi(15) } \\
2010\end{array}$ & $\begin{array}{l}1-590 \text { vaka } \\
591-760 \text { vaka } \\
761-1181 \text { vaka }\end{array}$ & Prospektif & $\begin{array}{l}0-1 \text { güvenlik } \\
\text { pedi }\end{array}$ & Belirtilmemiş & $\begin{array}{l}62 \\
84 \\
82\end{array}$ & $\begin{array}{l}82 \\
88 \\
90\end{array}$ & $\begin{array}{l}88 \\
93 \\
96\end{array}$ & - \\
\hline
\end{tabular}

$\mathrm{PB}=$ Prostat boyutu, VKİ=Vücut kitle indeksi

oranları \%69-96 arasındadır. 0-1 koruyucu ped kullanımını temel alan çalışmalarda 12 ay sonunda kontinans oranları ise \%89-92.5 arasındadır. En uzun takip süresi 36 ay olan Murphy ve ark.'larının çalışmasında kontinans oranı \%95 olarak bildirilmiştir (14). Tablo 2' de bazı komplike vakalardaki ve cerrahi tecrübe farklılıklarındaki kontinans oranları özetlenmiştir.

Prostatın boyutu $(12,16)$, vücut kitle indeksi $(5,17)$ ve cerrahın tecrübesi $(15,18)$ kontinans sağlanmasını etkileyebilen olası nedenlerdir. Hasta yaşı $(5,6,19,20)$, hastanın mevcut komorbiditeleri (7), alt üriner sistem semptomlarının şiddeti ve ameliyat öncesi erektil disfonksiyon varlığ 1 (5,8); RYLP geçiren hastalardaki kontinansı doğru orantılı olarak bozan diğer anlamlı faktörler olarak bildirilmiştir.

\section{RYLP Sonrası Kontinansı Sağlamaya Etki Eden Cerrahi Yaklaşımlar}

RYLP'nin daha yaygin olarak kullanılması ile sinir koruyucu cerrahi, anterior ve posterior rekonstrüksiyon, mesane boynu koruyucu yaklaşım gibi cerrahi teknikler geliştirilerek idrar kontinansının hem daha erken dönemde; hem de daha yüksek oranda sağlandığı göze çarpmaktadır.

Chung ve ark.'ları yaptıkları retrospektif karşılaştırmalı çalışmada, ekstraperitonal RYLP'de transperitoneal tekniğe göre 3., 6. ve 12. aylarda daha yüksek kontinans oranları rapor edilmiştir (21). Ancak ekstraperitoneal RYLP daha detaylı değerlendirilmesi için daha kapsamlı ve kanıt düzeyi yüksek çalışmalara ihtiyaç olduğu belirtilmelidir.

Freire ve ark.'larının yaptıkları ve 348 hastanın değerlendirildiği bir çalışmada mesane boynu koruyucu yaklaşımın 3. ve 6. ayda kontinansı sağlamada, mesane boynu korunmayan vakalara göre anlamlı olarak daha iyi sonuçlar sağladığ bildirilmektedir. Yirmidördüncü ayda ise; sonuçlar, mesane boynu koruyucu grupta \%100 olmakla beraber, aradaki fark istatistiksel olarak anlamlı değildir (22). Friedlander ve ark.'ları, yaptıkları bir prospektif çalışmada mesane boynu koruyucu grupta mesane boynu korunmayan (standart) gruba göre daha yüksek kontinans oranları bildirmişlerdir (23). Lee ve ark.'ları ise; yaptıkları bir çalışmada mesane boynu koruyucu cerrahi yapilan hastalarda erken dönemde (3. ay) kontinans düzeyini anlamlı olarak yüksek bulmuşlar, 1. y1lda ise; mesane boynu korunmayan hastalarla anlamlı fark bildirmemişlerdir (24). Erken dönemde tam kontinansın sağlanması açısından mesane koruyucu yaklaşımın faydalı olduğu görülmekte olup, daha geniş serilerle bu kanı desteklenmelidir.

Finley ve ark.'ları soğuk irrigasyon ve endorektal soğutucu balon ile sinir koruyucu RYLP yapilan hastaları standart cerrahi uygulanan grup ile karşılaştırmışlar ve soğutma yap1lan hastalarda anlamlı olarak daha yüksek erken kontinans oranları bildirmişlerdir (5). Lei ve ark.'ları atermal olarak dorsal venöz kompleksinin kesilmesi yöntemi ile yaptıkları çalışmada, 6. ayda hastalarında anlamlı olarak daha yüksek kontinans oranları bildirilmişler, ancak 12. ayda kontinans açısından aradaki fark istatistiksel olarak anlamlı saptanmamiştır (25).

Sammon ve ark.'ları üretrovezikal anastomozda V-loc (Covidien, Mansfield, MA) dikiş materyali ile standart monofilaman dikiş yöntemlerini randomize olarak karşılaştırmış ve anastomoz süresini kısaltması dışında kontinans açısından fark gösterememişlerdir (26). Zorn ve ark.'larının çalışmasında da benzer sonuçlar bildirilmiştir (27). Tablo 3'de RYLP sonrası kontinansı sağlamaya etki eden bazı cerrahi yaklaşımlar özetlenmiştir.

\section{Posterior Rekonstrüksiyon}

Posterior rekonstrüksiyon, vezikoüretral anastomoz tamamlanmadan önce rabdosfinkterin posterior tabakasının Denonvillier fasyası ve mesane arka yüzüne dikilmesi; yaklaştırılmasıdır. Bu sayede anastomoz gerginliği azaltılır ve mesane boynuna destek sağlanır. Posterior rekonstrüksiyonun RYLP'de kontinansa olası etkileri çok sayıda karşılaştırmalı çalışma ile değerlendirilmiştir. Tablo 4, bu çalışmaların özetini içermektedir. 
Tablo 3: RYLP sonrası kontinansı sağlamaya etki eden cerrahi yaklaşımlar

\begin{tabular}{|c|c|c|c|c|c|c|c|c|}
\hline $\begin{array}{l}\text { Yazar/ } \\
\text { Çalışma yılı }\end{array}$ & $\begin{array}{l}\text { Cerrahi yöntem/ } \\
\text { Vaka sayısı }\end{array}$ & $\begin{array}{l}\text { Çalışma } \\
\text { dizaynı }\end{array}$ & $\begin{array}{l}\text { Kontinans } \\
\text { tanımlama }\end{array}$ & Bilgi & $\begin{array}{l}\text { 1-3. ay } \\
\text { kontinans } \\
(\%)\end{array}$ & $\begin{array}{l}\text { 6. ay } \\
\text { kontinans } \\
(\%)\end{array}$ & $\begin{array}{l}\text { 12. ay } \\
\text { kontinans } \\
(\%)\end{array}$ & $\begin{array}{l}24-36 . \text { ay } \\
\text { kontinans } \\
(\%)\end{array}$ \\
\hline $\begin{array}{l}\text { Chung(21) } \\
2011\end{array}$ & $\begin{array}{l}\text { Transperitoneal, } \\
105 \\
\text { Ekstraperitoneal, } \\
155\end{array}$ & $\begin{array}{l}\text { Retrospektif } \\
\text { karşılaştırmalı }\end{array}$ & 0 ped & $\begin{array}{l}\text { Valide } \\
\text { anket }\end{array}$ & $\begin{array}{l}90 \\
92\end{array}$ & $\begin{array}{l}95 \\
97\end{array}$ & $\begin{array}{l}97 \\
99\end{array}$ & - \\
\hline $\begin{array}{l}\text { Freire(22) } \\
2009\end{array}$ & $\begin{array}{l}\text { Mesane boynu } \\
\text { koruyucu,348 } \\
\text { Standart* }^{*}, 271\end{array}$ & $\begin{array}{l}\text { Prospektif } \\
\text { karşılaştırmalı }\end{array}$ & 0 ped & $\begin{array}{l}\text { Valide } \\
\text { anket }\end{array}$ & $\begin{array}{l}66 \\
26\end{array}$ & - & $\begin{array}{l}86 \\
81\end{array}$ & $\begin{array}{l}100 \\
96\end{array}$ \\
\hline $\begin{array}{l}\text { Freidlander(23) } \\
2012\end{array}$ & $\begin{array}{l}\text { Mesane boynu } \\
\text { koruyucu,791 } \\
\text { Standart,276 }\end{array}$ & Prospektif & $\begin{array}{l}0-1 \text { güvenlik } \\
\text { pedi }\end{array}$ & $\begin{array}{l}\text { Valide } \\
\text { anket }\end{array}$ & - & $\begin{array}{l}80 \\
63\end{array}$ & - & $\begin{array}{l}86 \\
75\end{array}$ \\
\hline $\begin{array}{l}\text { Lei(25) } \\
2011\end{array}$ & $\begin{array}{l}\text { Selektif sütür,240 } \\
\text { Standart,303 }\end{array}$ & $\begin{array}{l}\text { Prospektif } \\
\text { karşılaştırmalı }\end{array}$ & 0 ped & $\begin{array}{l}\text { Valide } \\
\text { anket }\end{array}$ & - & $\begin{array}{l}61 \\
40\end{array}$ & $\begin{array}{l}70 \\
74\end{array}$ & - \\
\hline $\begin{array}{l}\text { Sammon(26) } \\
2011\end{array}$ & $\begin{array}{l}\text { Balıksırtı dikiş } \\
\text { (V-loc), } 33 \\
\text { Standart, } 31\end{array}$ & $\begin{array}{l}\text { Randomize } \\
\text { Kontrollü }\end{array}$ & 0 ped & $\begin{array}{l}\text { Valide } \\
\text { anket }\end{array}$ & $\begin{array}{l}51 \\
50\end{array}$ & - & - & - \\
\hline $\begin{array}{l}\text { Zorn(27) } \\
2012\end{array}$ & $\begin{array}{l}\text { Balıksırtı dikiş } \\
\text { (V-loc), } 35 \\
\text { Standart, } 25\end{array}$ & $\begin{array}{l}\text { Randomize } \\
\text { kontrollü }\end{array}$ & 0 ped & $\begin{array}{l}\text { Valide } \\
\text { anket }\end{array}$ & $\begin{array}{l}69 \\
64\end{array}$ & $\begin{array}{l}92 \\
88\end{array}$ & - & - \\
\hline
\end{tabular}

*Standart: İlgili cerrahi teknik uygulanmayan grup

Tablo 4: RYLP'de posterior (arka) rekonstrüksiyonun kontinansa etkilerini karşılaştıran çalışmalar

\begin{tabular}{|c|c|c|c|c|c|c|c|}
\hline $\begin{array}{l}\text { Yazar/ } \\
\text { Çalışma yılı }\end{array}$ & $\begin{array}{l}\text { Cerrahi yöntem/ } \\
\text { Vaka sayısı }\end{array}$ & $\begin{array}{l}\text { Çalışma } \\
\text { dizaynı }\end{array}$ & $\begin{array}{l}\text { Kontinans } \\
\text { tanımlama }\end{array}$ & $\begin{array}{l}\text { Bilgi } \\
\text { toplama }\end{array}$ & $\begin{array}{l}\text { 1. ay } \\
\text { kontinans } \\
(\%)\end{array}$ & $\begin{array}{l}\text { 3. ay } \\
\text { kontinans } \\
(\%)\end{array}$ & $\begin{array}{l}\text { 6. ay } \\
\text { kontinans } \\
(\%)\end{array}$ \\
\hline $\begin{array}{l}\text { Krane(28) } \\
2009\end{array}$ & $\begin{array}{l}\text { Arka } R, 42 \\
\text { Standart }{ }^{*}, 42\end{array}$ & $\begin{array}{l}\text { Retrospektif } \\
\text { karşılaştırmalı }\end{array}$ & $\begin{array}{l}0-1 \text { güvenlik } \\
\text { pedi }\end{array}$ & Görüşme & $\begin{array}{l}85 \\
86\end{array}$ & - & - \\
\hline $\begin{array}{l}\text { Woo(29) } \\
2009\end{array}$ & $\begin{array}{l}\text { Arka R,69 } \\
\text { Standart,63 }\end{array}$ & $\begin{array}{l}\text { Prospektif } \\
\text { karşılaştırmalı }\end{array}$ & $\begin{array}{l}0-1 \text { güvenlik } \\
\text { pedi }\end{array}$ & $\begin{array}{l}\text { Valide } \\
\text { anket }\end{array}$ & \multicolumn{3}{|c|}{$\begin{array}{l}\text { Ort. kontinansa kadar süre } 90 \text { gün } \\
\text { Ort. kontinansa kadar süre } 150 \text { gün }\end{array}$} \\
\hline $\begin{array}{l}\text { Joshi(30) } \\
2010\end{array}$ & $\begin{array}{l}\text { Arka R,53 } \\
\text { Standart,54 }\end{array}$ & $\begin{array}{l}\text { Prospektif } \\
\text { karşılaştırmalı }\end{array}$ & 0 ped & $\begin{array}{l}\text { Valide } \\
\text { anket }\end{array}$ & - & $\begin{array}{l}52 \\
63\end{array}$ & $\begin{array}{l}76 \\
84\end{array}$ \\
\hline $\begin{array}{l}\operatorname{Kim}(31) \\
2010\end{array}$ & $\begin{array}{l}\text { Arka R,25 } \\
\text { Standart,25 }\end{array}$ & $\begin{array}{l}\text { Retrospektif } \\
\text { karşılaştırmalı }\end{array}$ & 0 ped & $\begin{array}{l}\text { Valide } \\
\text { anket }\end{array}$ & $\begin{array}{l}76 \\
71\end{array}$ & $\begin{array}{l}88 \\
80\end{array}$ & $\begin{array}{l}96 \\
96\end{array}$ \\
\hline $\begin{array}{l}\text { Coelho(32) } \\
2011\end{array}$ & $\begin{array}{l}\text { Arka R,473 } \\
\text { Standart,330 }\end{array}$ & $\begin{array}{l}\text { Prospektif } \\
\text { karşılaştırmalı }\end{array}$ & 0 ped & $\begin{array}{l}\text { Valide } \\
\text { anket }\end{array}$ & $\begin{array}{l}51 \\
42\end{array}$ & $\begin{array}{l}91 \\
92\end{array}$ & $\begin{array}{l}97 \\
96\end{array}$ \\
\hline $\begin{array}{l}\text { Sutherland(33) } \\
2011\end{array}$ & $\begin{array}{l}\text { Arka R,47 } \\
\text { Standart,47 }\end{array}$ & $\begin{array}{l}\text { Randomize } \\
\text { kontrollü }\end{array}$ & $\begin{array}{l}0-1 \text { güvenlik } \\
\text { pedi }\end{array}$ & $\begin{array}{l}\text { Valide } \\
\text { anket }\end{array}$ & - & $\begin{array}{l}63 \\
81\end{array}$ & - \\
\hline $\begin{array}{l}\text { Jeong(34) } \\
2012\end{array}$ & $\begin{array}{l}\text { Arka R,116 } \\
\text { Standart,126 }\end{array}$ & $\begin{array}{l}\text { Retrospektif } \\
\text { karşılaştırmalı }\end{array}$ & 0 ped & $\begin{array}{l}\text { Valide } \\
\text { anket }\end{array}$ & $\begin{array}{l}58 \\
45\end{array}$ & $\begin{array}{l}82 \\
70\end{array}$ & $\begin{array}{l}95 \\
86\end{array}$ \\
\hline
\end{tabular}

*Standart: Posterior rekonstrüksiyon yapılmayan grup

Tablo 4'deki çalışmalara bakıldığında posterior rekonstrüksiyon yapılan olgularda erken dönemde (1. ay) kontinans oranları daha yüksek olarak bildirilmiştir ancak 3. ve 6. ay oranlarında genellikle anlamlı derecede fark rapor edilmemiştir. Jeong ve ark. çalışmasında ise 1., 3. ve 6. aylarda posterior rekonstrüksiyon uygulaması, rekonstrüksiyon uygulanmayan standart gruba göre anlamlı olarak daha yüksek kontinans oranları bildirilmektedir (34).

\section{Posterior ve Anterior Rekonstrüksiyon}

Posterior rekonstrüksiyon ile birlikte anterior (ön) rekonstrüksiyon yapılmasıyla kontinans oranlarının artacağına dair çalışmalar bulunmaktadır. Anterior rekonstrüksiyonda rabdosfinkter puboprostatik ligamanlarla beraber pubik periosta asılır. Anterior ve posterior rekonstrüksiyonun birlikte yapılması total rekonstrüksiyon olarak da adlandırılmaktadır. Tablo 5'te bu çalışmaların özeti verilmektedir.

Total rekonstrüksiyonla ilgili randomize kontrollü çalışmaların aynı seriyi içeren ikisinde standart yöntemle aralarında fark gösterilememiştir $(35,36)$. Buna karşın Koliakos ve Hurtes' in çalışmalarında total rekonstrüksiyon ile daha yüksek kontinans oranları bildirilmiştir $(37,39)$. Tan ve ark.'larının 1600 hastayı içeren serilerinde total rekonstrüksiyon grubunda anlamlı avantaj sağlandığı gösterilmiştir (19). Atuğ ve ark.'larının prospektif çalışmasında da; erken 
Tablo 5: RYLP'de total onarım ile standart yöntemin karşılaştırılması

\begin{tabular}{|c|c|c|c|c|c|c|c|c|}
\hline $\begin{array}{l}\text { Yazar/ } \\
\text { Çalışma yılı }\end{array}$ & $\begin{array}{l}\text { Cerrahi yöntem/ } \\
\text { Vaka sayısı }\end{array}$ & $\begin{array}{l}\text { Çalışma } \\
\text { dizaynı }\end{array}$ & $\begin{array}{l}\text { Kontinans } \\
\text { tanımlama }\end{array}$ & $\begin{array}{l}\text { Bilgi } \\
\text { toplama }\end{array}$ & $\begin{array}{l}\text { 1. ay } \\
\text { kontinans } \\
(\%)\end{array}$ & $\begin{array}{l}\text { 3. ay } \\
\text { kontinans } \\
(\%)\end{array}$ & $\begin{array}{l}\text { 6. ay } \\
\text { kontinans } \\
(\%)\end{array}$ & $\begin{array}{l}\text { 12-24. ay } \\
\text { kontinans } \\
(\%)\end{array}$ \\
\hline $\begin{array}{l}\text { Menon(35) } \\
2008\end{array}$ & $\begin{array}{l}\text { Total onarım,59 } \\
\text { Standart, } 57\end{array}$ & $\begin{array}{l}\text { Randomize } \\
\text { kontrollü }\end{array}$ & $\begin{array}{l}0-1 \text { güvenlik } \\
\text { pedi }\end{array}$ & $\begin{array}{l}\text { Valide } \\
\text { anket }\end{array}$ & $\begin{array}{l}80 \\
74\end{array}$ & - & - & - \\
\hline $\begin{array}{l}\text { Sammon(36) } \\
2010\end{array}$ & $\begin{array}{l}\text { Total onarım, } 46 \\
\text { Standart, } 50\end{array}$ & $\begin{array}{l}\text { Randomize } \\
\text { kontrollü }\end{array}$ & 0 ped & $\begin{array}{l}\text { Valide } \\
\text { anket }\end{array}$ & $\begin{array}{l}42 \\
47\end{array}$ & - & - & $\begin{array}{l}82 \\
80\end{array}$ \\
\hline $\begin{array}{l}\text { Koliakos(37) } \\
2010\end{array}$ & $\begin{array}{l}\text { Total onarım,24 } \\
\text { Standart,26 }\end{array}$ & $\begin{array}{l}\text { Randomize } \\
\text { kontrollü }\end{array}$ & $\begin{array}{l}0-1 \text { güvenlik } \\
\text { pedi }\end{array}$ & $\begin{array}{l}\text { Valide } \\
\text { anket }\end{array}$ & $\begin{array}{l}65 \\
33\end{array}$ & - & - & - \\
\hline $\begin{array}{l}\text { Atuğ(38) } \\
2012\end{array}$ & $\begin{array}{l}\text { Total onarim,125 } \\
\text { Standart,120 }\end{array}$ & $\begin{array}{l}\text { Prospektif } \\
\text { karşılaştırmalı }\end{array}$ & 0 ped & $\begin{array}{l}\text { Valide } \\
\text { anket }\end{array}$ & $\begin{array}{l}72 \\
49\end{array}$ & $\begin{array}{l}80 \\
76\end{array}$ & $\begin{array}{l}84 \\
80\end{array}$ & $\begin{array}{l}91 \\
88\end{array}$ \\
\hline $\begin{array}{l}\text { Hurtes(39) } \\
2012\end{array}$ & $\begin{array}{l}\text { Total onarım,39 } \\
\text { Standart,33 }\end{array}$ & $\begin{array}{l}\text { Randomize } \\
\text { kontrollü }\end{array}$ & 0 ped & $\begin{array}{l}\text { Valide } \\
\text { anket }\end{array}$ & $\begin{array}{l}27 \\
7\end{array}$ & $\begin{array}{l}45 \\
15\end{array}$ & $\begin{array}{l}65 \\
58\end{array}$ & - \\
\hline $\begin{array}{l}\operatorname{Tan}(19) \\
2010\end{array}$ & $\begin{array}{l}\text { Total onarım,1383 } \\
\text { Ön onarım,303 } \\
\text { Standart,214 }\end{array}$ & $\begin{array}{l}\text { Prospektif } \\
\text { karşılaştırmalı }\end{array}$ & $\begin{array}{l}0-1 \text { güvenlik } \\
\text { pedi }\end{array}$ & $\begin{array}{l}\text { Valide } \\
\text { anket }\end{array}$ & - & $\begin{array}{l}91 \\
77 \\
50\end{array}$ & $\begin{array}{l}95 \\
86 \\
62\end{array}$ & $\begin{array}{l}98 \\
92 \\
82\end{array}$ \\
\hline
\end{tabular}

Standart: Herhangi bir rekonstrüksiyon yapılmayan grup

dönemde total rekonstrüksiyonun avantaj sağladığı; ancak uzun dönemde kontinans oranlarının benzer olduğu bildirilmiştir (38).

Tewari ve ark.'ları total rekonstrüksiyonun yanında apikal uretranın çepeçevre diseksiyonu, detrusor trigonoplasti ve suprapubik tüp konmasıyla birleştirdikleri teknikleri ile postoperatif 6 . haftada $\% 60.9$ oranında tam kontinans oranı bildirmişlerdir (40).

Postprostatektomik inkontinans durumunda hastalara pelvik taban egzersizleri kontinansın erken sağlanması için genellikle önerilmektedir. Geraets ve ark.'larının çalışmasinda ise; operasyon öncesi ve sonrası pelvik taban kas egzersizlerinin kontinans üzerine anlamlı katkı sağlamadığ bildirilmiştir (41).

Sammon ve ark.'ları RYLP sonrası erken kontinans kazanılmasında, sinir koruyucu cerrahi yapılmasının ve operasyon sonrası mesane boşaltımı için suprapubik tüp yerleştirilmesinin birbirinden bağımsız anlamlı göstergeler olduğunu belirtmişlerdir (42).

Srivastava ve ark.'ları yaptıkları çalışmada sinir koruyucu yaklaşım yapılarak hamak yapısı korunmasının erken kontinans sağlama açısından bağımsız bir öngörücü olduğunu rapor etmişlerdir (43). Hamada ve ark.'ları ise; maksimum uretra boyunun korunmasinin posterior ve anterior rekontrüksiyon ile beraber kontinans oranlarını artırdığını bildirmişlerdir (44).

Kojima ve ark.'ları operasyon esnasında mesane boynunu pubik kemiğe sabitleyen dikiş ile sling (45) tekniğini 27 hastada uygulamışlar ve uygulanmayan 30 hastaya göre anlamlı erken kontinans oranları bildirmişlerdir.

Yukarıda bahsettiğimiz umut vaad eden tekniklerin geniş serileri ve uzun dönem sonuçlarını beklemek gerekmektedir.

Jeong ve ark.'ları kontinans açısından nomogram geliştirmek için yaptıkları bir çalışmada, RYLP ile ARP vakaları randomize edilerek karşılaştırmışlardır. Multivarite analizinde RYLP, genç yaş ve membranoz uretra'nın uzunluğu 1., 3. ve 12. ay kontinans iyileşmesi açısından anlamlı öngörücüler olarak bulunurken; prostat boyutu ve sinir koruyucu yaklaşım sadece 12. ay kontinans iyileşmesinde anlamlı öngörücü olarak bulunmuştur (46).

\section{Sonuç}

RYLP, kontinans açısından ARP ve LRP ile karşılaştırıldığında günümüzde RYLP lehine anlamlı avantajlı sonuçlar rapor edilmektedir (2). RYLP'de idrar kaçırma oranları operasyon öncesi hasta özellikleri, cerrah tecrübesi, cerrahi teknik ve kontinans tanımı için kullanılan metodolojiden etkilenmektedir. Hasta yaşı, hastanın vücut kitle indeksi, mevcut komorbiditeleri, alt üriner sistem şikayetlerinin varlığı ve prostat boyutu, RYLP sonrası kontinans için olası öngürücü etkenlerdir. Operasyon öncesi bu özellikler nedeniyle hastaya dikkatlice bilgi verilmelidir. Çünkü hastanın RYLP sonrası yaşam kalitesini etkileyen en önemli etken kontinans durumudur ve erken tam kontinans hasta memnuniyetini artırmaktadır. RYLP ile günümüze kadar olan çalışmalarda, geliştirilen cerrahi teknik (özellikle sinir koruyucu, posterior onarım, total onarım, mesane boynu koruyucu yaklaşım gibi) yaklaşımlarla tam kontinans daha erken sağlanabilmektedir.

Biz, kendi hasta grubumuzdan edindiğimiz tecrübe ile birlikte; uygun hasta grubunda sinir koruyucu cerrahi ile beraber posterior ve anterior onarımin yapılması, olabildiğince uzun uretra boyutunun sağlanması ve mümkünse mesane boynunun en yüksek düzeyde, 360 derece korunmasını tam kontinans sağlanması açısından özellikle önermekteyiz.

\section{Kaynaklar}

1. van der Poel HG, Tillier C, de Blok WM, Acar C, van Muilekom EH, van den Bergh RC. Interview-based versus questionnaire-based quality of life outcomes before and after prostatectomy. J Endourol 2013 Nov;27(11):1411-6.

2. Ficarra V, Novara G, Rosen RC, Artibani W, Carroll PR, Costello A, Menon M, Montorsi F, Patel VR, Stolzenburg J-U. Systematic review and meta-analysis of studies reporting urinary continence recovery after robot-assisted radical prostatectomy. European Urology 2012;62(3):40517. 
3. Ficarra V, Novara G, Artibani W, Cestari A, Galfano A, Graefen M, Guazzoni G, Guillonneau B, Menon M, Montorsi F. Retropubic, laparoscopic, and robotassisted radical prostatectomy: a systematic review and cumulative analysis of comparative studies. European urology 2009;55(5):1037-63.

4. Guillonneau B, Cathelineau X, Barret E, Rozet F, Vallancien G. Laparoscopic radical prostatectomy: technical and early oncological assessment of 40 operations. Eur Urol 1999;36(1):14-20.

5. Finley DS, Osann K, Chang A, Santos R, Skarecky D, Ahlering TE. Hypothermic robotic radical prostatectomy: impact on continence. J Endourol 2009 Sep;23(9):1443-50.

6. Lee DJ, Cheetham P, Badani KK. Predictors of early urinary continence after robotic prostatectomy. Can J Urol 2010 Jun;17(3):5200-5.

7. Novara G, Ficarra V, D'Elia C, Secco S, Cioffi A, Cavalleri $S$, Artibani W. Evaluating urinary continence and preoperative predictors of urinary continence after robot assisted laparoscopic radical prostatectomy. J Urol 2010 Sep;184(3):1028-33.

8. Shikanov S, Desai V, Razmaria A, Zagaja GP, Shalhav AL. Robotic radical prostatectomy for elderly patients: probability of achieving continence and potency 1 year after surgery. J Urol 2010 May;183(5):1803-7.

9. Martin AD, Nakamura LY, Nunez RN, Wolter CE, Humphreys MR, Castle EP. Incontinence after radical prostatectomy: a patient centered analysis and implications for preoperative counseling. J Urol 2011 Jul;186(1):204-8.

10. Patel VR, Sivaraman A, Coelho RF, Chauhan S, Palmer KJ, Orvieto MA, Camacho I, Coughlin G, Rocco B. Pentafecta: a new concept for reporting outcomes of robot-assisted laparoscopic radical prostatectomy. Eur Urol 2011 May;59(5):702-7.

11. Xylinas E, Ploussard G, Durand X, de La Taille A, Gillion N, Allory Y, Vordos D, Hoznek A, Abbou CC, Salomon L. Evaluation of combined oncological and functional outcomes after radical prostatectomy: trifecta rate of achieving continence, potency and cancer control-a literature review. Urology 2010 Nov;76(5):1194-8.

12. Link BA, Nelson R, Josephson DY, Yoshida JS, Crocitto LE, Kawachi MH, Wilson TG. The impact of prostate gland weight in robot assisted laparoscopic radical prostatectomy. J Urol 2008 Sep;180(3):928-32.

13. Greco KA, Meeks JJ, Wu S, Nadler RB. Robot-assisted radical prostatectomy in men aged $>$ or $=70$ years. BJU Int 2009 Nov;104(10):1492-5.

14. Murphy DG, Kerger M, Crowe H, Peters JS, Costello AJ. Operative details and oncological and functional outcome of robotic-assisted laparoscopic radical prostatectomy: 400 cases with a minimum of 12 months follow-up. European Urology 2009;55(6):1358-67.

15. Samadi DB, Muntner P, Nabizada-Pace F, Brajtbord JS, Carlucci J, Lavery HJ. Improvements in robot-assisted prostatectomy: the effect of surgeon experience and technical changes on oncologic and functional outcomes. J Endourol 2010 Jul;24(7):1105-10.

16. Skolarus TA, Hedgepeth RC, Zhang Y, Weizer AZ, Montgomery JS, Miller DC, Wood DP, Jr., Hollenbeck BK. Does robotic technology mitigate the challenges of large prostate size? Urology 2010 Nov;76(5):1117-21.
17. Wiltz AL, Shikanov S, Eggener SE, Katz MH, Thong AE, Steinberg GD, Shalhav AL, Zagaja GP, Zorn KC. Robotic radical prostatectomy in overweight and obese patients: oncological and validated-functional outcomes. Urology 2009 Feb;73(2):316-22.

18. Zorn KC, Wille MA, Thong AE, Katz MH, Shikanov SA, Razmaria A, Gofrit ON, Zagaja GP, Shalhav AL. Continued improvement of perioperative, pathological and continence outcomes during 700 robot-assisted radical prostatectomies. Can J Urol 2009 Aug;16(4):47429.

19. Tan G, Srivastava A, Grover S, Peters D, Dorsey P, Jr., Scott A, Jhaveri J, Tilki D, Te A, Tewari A. Optimizing vesicourethral anastomosis healing after robot-assisted laparoscopic radical prostatectomy: lessons learned from three techniques in 1900 patients. J Endourol 2010 Dec;24(12):1975-83.

20. Jeong SJ, Yi J, Chung MS, Kim DS, Lee WK, Park H, Yoon CY, Hong SK, Byun SS, Lee SE. Early recovery of urinary continence after radical prostatectomy: correlation with vesico-urethral anastomosis location in the pelvic cavity measured by postoperative cystography. Int J Urol 2011 Jun;18(6):444-51.

21. Chung JS, Kim WT, Ham WS, Yu HS, Chae Y, Chung SH, Choi YD. Comparison of oncological results, functional outcomes, and complications for transperitoneal versus extraperitoneal robot-assisted radical prostatectomy: a single surgeon's experience. J Endourol 2011 May;25(5):787-92.

22. Freire MP, Weinberg AC, Lei $Y$, Soukup JR, Lipsitz SR, Prasad SM, Korkes F, Lin T, Hu JC. Anatomic bladder neck preservation during robotic-assisted laparoscopic radical prostatectomy: description of technique and outcomes. Eur Urol 2009 Dec;56(6):972-80.

23. Friedlander DF, Alemozaffar M, Hevelone ND, Lipsitz SR, Hu JC. Stepwise description and outcomes of bladder neck sparing during robot-assisted laparoscopic radical prostatectomy. J Urol 2012 Nov;188(5):1754-60.

24. Lee Z, Sehgal SS, Graves RV, Su YK, Llukani E, Monahan K, McGill A, Eun D, Lee DI. Functional and oncologic outcomes of graded bladder neck preservation during robot-assisted radical prostatectomy. J Endourol2014 Jan;28(1):48-55.

25. Lei Y, Alemozaffar M, Williams SB, Hevelone N, Lipsitz SR, Plaster BA, Amarasekera CA, Ulmer WD, Huang AC, Kowalczyk KJ, Hu JC. Athermal division and selective suture ligation of the dorsal vein complex during robotassisted laparoscopic radical prostatectomy: description of technique and outcomes. Eur Urol 2011 Feb;59(2):23543.

26. Sammon J, Kim TK, Trinh QD, Bhandari A, Kaul S, Sukumar S, Rogers CG, Peabody JO. Anastomosis during robot-assisted radical prostatectomy: randomized controlled trial comparing barbed and standard monofilament suture. Urology 2011 Sep;78(3):572-9.

27. Zorn KC, Trinh QD, Jeldres C, Schmitges J, Widmer H, Lattouf JB, Sammon J, Liberman D, Sun M, Bianchi M, Karakiewicz PI, Denis R, Gautam G, El-Hakim A. Prospective randomized trial of barbed polyglyconate suture to facilitate vesico-urethral anastomosis during robot-assisted radical prostatectomy: time reduction and cost benefit. BJU Int 2012 May;109(10):1526-32. 
28. Krane LS, Wambi C, Bhandari A, Stricker HJ. Posterior support for urethrovesical anastomosis in robotic radical prostatectomy: single surgeon analysis. Can J Urol 2009 Oct;16(5):4836-40.

29. Woo JR, Shikanov S, Zorn KC, Shalhav AL, Zagaja GP. Impact of posterior rhabdosphincter reconstruction during robot-assisted radical prostatectomy: retrospective analysis of time to continence. J Endourol 2009 Dec;23(12):1995-9.

30. Joshi N, de Blok W, van Muilekom E, van der Poel $H$. Impact of posterior musculofascial reconstruction on early continence after robot-assisted laparoscopic radical prostatectomy: results of a prospective parallel group trial. Eur Urol 2010 Jul;58(1):84-9.

31. Kim IY, Hwang EA, Mmeje C, Ercolani M, Lee DH. Impact of posterior urethral plate repair on continence following robot-assisted laparoscopic radical prostatectomy. Yonsei Med J 2010 May;51(3):427-31.

32. Coelho RF, Chauhan S, Orvieto MA, Sivaraman A, Palmer KJ, Coughlin G, Patel VR. Influence of modified posterior reconstruction of the rhabdosphincter on early recovery of continence and anastomotic leakage rates after robot-assisted radical prostatectomy. Eur Urol 2011 Jan;59(1):72-80.

33. Sutherland DE, Linder B, Guzman AM, Hong M, Frazier HA, 2nd, Engel JD, Bianco FJ, Jr. Posterior rhabdosphincter reconstruction during robotic assisted radical prostatectomy: results from a phase II randomized clinical trial. J Urol 2011 Apr;185(4):1262-7.

34. Jeong CW, Oh JJ, Jeong SJ, Hong SK, Byun SS, Choe G, Lee SE. Novel posterior reconstruction technique during robot-assisted laparoscopic prostatectomy: description and comparative outcomes. Int J Urol 2012 Jul;19(7):683-7.

35. Menon M, Muhletaler F, Campos M, Peabody JO. Assessment of early continence after reconstruction of the periprostatic tissues in patients undergoing computer assisted (robotic) prostatectomy: results of a 2 group parallel randomized controlled trial. J Urol 2008 Sep;180(3):1018-23.

36. Sammon JD, Muhletaler F, Peabody JO, Diaz-Insua M, Satyanaryana R, Menon M. Long-term functional urinary outcomes comparing single- vs double-layer urethrovesical anastomosis: two-year follow-up of a twogroup parallel randomized controlled trial. Urology 2010 Nov;76(5):1102-7.

37. Koliakos N, Mottrie A, Buffi N, De Naeyer G, Willemsen $\mathrm{P}$, Fonteyne E. Posterior and anterior fixation of the urethra during robotic prostatectomy improves early continence rates. Scand J Urol Nephrol 2010 Feb;44(1):510 .

38. Atug F, Kural AR, Tufek I, Srivastav S, Akpinar H. Anterior and posterior reconstruction technique and its impact on early return of continence after robot-assisted radical prostatectomy. J Endourol 2012 Apr;26(4):381-6.

39. Hurtes X, Roupret M, Vaessen C, Pereira H, Faivre
d'Arcier B, Cormier L, Bruyere F. Anterior suspension combined with posterior reconstruction during robotassisted laparoscopic prostatectomy improves early return of urinary continence: a prospective randomized multicentre trial. BJU Int 2012 Sep;110(6):875-83.

40. Tewari AK, Ali A, Ghareeb G, Ludwig W, Metgud S, Theckumparampil N, Takenaka A, Chugtai B, Shrivastava A, Kaplan SA, Leung R, Paryani R, Grushow S, Durand M, Peyser A, Chopra S, Harneja N, Lee RK, Herman M, Robinson B, Shevchuck MM. Improving time to continence after robot-assisted laparoscopic prostatectomy: augmentation of the total anatomic reconstruction technique by adding dynamic detrusor cuff trigonoplasty and suprapubic tube placement. J Endourol 2012 Dec;26(12):1546-52.

41. Geraerts I, Van Poppel H, Devoogdt N, Joniau S, Van Cleynenbreugel B, De Groef A, Van Kampen M. Influence of preoperative and postoperative pelvic floor muscle training (PFMT) compared with postoperative PFMT on urinary incontinence after radical prostatectomy: a randomized controlled trial. Eur Urol 2013 Nov;64(5):766-72.

42. Sammon JD, Sharma P, Trinh QD, Ghani KR, Sukumar S, Menon M. Predictors of immediate continence following robot-assisted radical prostatectomy. J Endourol 2013 Apr;27(4):442-6.

43. Srivastava A, Chopra S, Pham A, Sooriakumaran P, Durand M, Chughtai B, Gruschow S, Peyser A, Harneja N, Leung R, Lee R, Herman M, Robinson B, Shevchuk $M$, Tewari A. Effect of a risk-stratified grade of nervesparing technique on early return of continence after robot-assisted laparoscopic radical prostatectomy. Eur Urol 2013 Mar;63(3):438-44.

44. Hamada A, Razdan S, Etafy MH, Fagin R. Early Return of Continence in Patients Undergoing Robot-Assisted Laparoscopic Prostatectomy Using Modified Maximal Urethral Length Preservation Technique. J Endourol 2014 Apr 16.

45. Kojima Y, Hamakawa T, Kubota Y, Ogawa S, Haga N, Tozawa K, Sasaki S, Hayashi Y, Kohri K. Bladder neck sling suspension during robot-assisted radical prostatectomy to improve early return of urinary continence: a comparative analysis. Urology 2014 Mar;83(3):632-9.

46. Jeong SJ, Yeon JS, Lee JK, Cha WH, Jeong JW, Lee BK, Lee SC, Jeong CW, Kim JH, Hong SK, Byun SS, Lee SE. Development and validation of nomograms to predict the recovery of urinary continence after radical prostatectomy: comparisons between immediate, early, and late continence. World J Urol 2014 Apr;32(2):437-44.

Yazışma Adresi:

Fatih Atuğ

Istanbul Gayrettepe Florence Nightingale Hastanesi, Cemil Aslan Güder Sok. No: 8 Gayrettepe, Istanbul

Tel:+902122883400

e-mail: fatihatug@gmail.com 